\title{
A Validated RP-HPLC Method for the Simultaneous Estimation of Atazanavir and Ritonavir in Pharmaceutical Dosage Forms
}

\author{
M.Sathish Kumar, B.Sandhya Rani, N.Mounika, J.Mamatha, J.Kranthi Kumar.
}

1)Kvk College of Pharmacy, Surmaiguda, Rangareddy, Hyderabad, India.

2) Mewar University, Chittograh, Rajasthan

Corresponding author Email: meruvasathish84@gmail.com

\begin{abstract}
A rapid, precise and accurate reverse phase high performance liquid chromatographic method have been developed for the validated of Atazanavir and Ritonavir, in its pure form as well as in tablet dosage form.

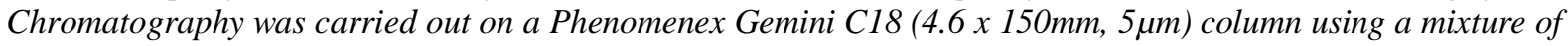
Methanol: Water $(90: 10 \% \mathrm{v} / \mathrm{v})$ as the mobile phase at a flow rate of $1.0 \mathrm{ml} / \mathrm{min}$, the detection was carried out at $249 \mathrm{~nm}$. The retention time of the Ritonavir and Atazanavir was 2.256, $5.427 \pm 0.02 \mathrm{~min}$ respectively. The method produce linearity responses in the range of $5-25 \mathrm{mg} / \mathrm{ml}$ of Ritonavir and $15-75 \mathrm{mg} / \mathrm{ml}$ of Atazanavir. The method precision for the determination of assay was below 2.0\%RSD. The method is useful for the quality and quality control of bulk and pharmaceutical formulations.
\end{abstract}

Keywords: Atazanavir, Ritonavir, RP-HPLC, validation.

\section{INTRODUCTION}

Atazanavir Sulphate Methyl is a Antiretroviral drug N- [(1S)-1-\{ [(2S,3S) - 3 - hydroxy-4- [(2S)-2[(methoxycarbonyl) amino] - 3, 3 - dimethyl - $\mathrm{N}^{\prime}-\quad\{[4-($ pyridin-2-yl)phenyl $]$ methyl $\}$ butanehydrazido]-1- phenylbutan-2-yl] carbamoyl -2, 2 - dimethylpropyl] carbamate sulphate is a azapeptide HIV-1 protease inhibitor The compound selectively inhibits the virusspecific processing of viral Gag and Gag-Pol polyproteins in HIV-1 infected cells, thus preventing formation of mature virions. Ritonavir is a Antiretroviral drug 1,3-thiazol- 5-ylmethyl N-[(2S,3S,5S)-3-hydroxy-5-[(2S)-3methyl-2 \{[methyl(\{[2-(propan-2-yl)-1,3-tiazole-4- yl]methyl $\})$ carbamoyl]amino $\}$ butanamido]-1,6diphenylhexan-2-yl] carbamate. Ritonavir inhibits the HIV viral protease enzyme. This prevents cleavage of the gag-pol polyprotein and, therefore, improper viral assembly results. This subsequently results in noninfectious, immature viral particles. Literature survey revealed that very few methods have been reported for the analysis of Atazanavir and Ritonavir combinational dosage forms which include UV spectroscopy, Reverse Phase High performance Liquid Chromatography, Densitometric method, HPTLC methods

\section{EXPERIMENTAL}

\section{Reagents and Chemicals}

Ritonavir API and atanavir API were obtained as gift sample from sura labs. Acetonitrile,Water, methanol is used of HPLC grade and purchased

\section{Instrumentation}

Chromatographic separation was performed on a Waters HPLC with auto sampler and PDA Detector 996.variablewavelength programmable UV/VIS detector, Phenomenex Gemini C18 $(4.6 \times 150 \mathrm{~mm}, 5 \mu)$ with10 $\mu$ l fixed loop.

\section{Chromatographic conditions}

Phenomenex Gemini C18 $(4.6 \times 150 \mathrm{~mm}, 5 \mu)$ were the column used for separation. Mobile phase

Containing a mixture of Methanol: Water (90:10\% v/v) in $1000 \mathrm{ml}$ of water and $\mathrm{pH}$ was adjusted to 4.2 in the ratio $50: 50 \mathrm{v} / \mathrm{v}$ was delivered at a flow rate of $1.0 \mathrm{ml} / \mathrm{min}$ with detection at $249 \mathrm{~nm}$. The mobile phase is filtered through a 0.45 nylon filter and sonicated for $20 \mathrm{~min}$. 


\section{Method development}

Acetonitrile, methanol and water in different proportions were tried and finally Methanol: Water = $90: 10 \mathrm{v} / \mathrm{v}$ was selected appropriate mobile phase which gave good resolution, retention time and acceptable system suitability parameters.

\section{Procedure}

\section{Preparation of standard solution}

Accurately weighed and transfer $10 \mathrm{mg}$ of Ritonavir and Atazanavir working standard into a $10 \mathrm{ml}$ of dry volumetric flasks add about $7 \mathrm{ml}$ of Methanol and $3 \mathrm{ml}$ of water sonicate to dissolve and removal of air completely and make volume up to the mark with the same Methanol and water.

Further pipette $0.15 \mathrm{ml}$ of the above Ritonavir and $0.45 \mathrm{ml}$ of Atazanavir stock solutions into a $10 \mathrm{ml}$ volumetric flask and dilute up to the mark with Methanol.

\section{Procedure:}

Inject the samples to the Rp-Hplc by changing the chromatographic conditions and record the chromatograms, note the conditions of proper peak elution and retention for performing validation parameters as per ICH guidelines

\section{Linearity}

Accurately measured volumes of working standard solution of Ritonavir and Atazanavir was transferred into a series of $10 \mathrm{ml}$ volumetricflasks and diluted appropriately with mobilephase. $10 \mu \mathrm{l}$ of each solution was injected under chromatographic conditions described above. Calibration curves were obtained by plotting the response (area of drug peak) versus concentration of drug. Regression were calculated. The method was found linear over a concentration range of $5-25 \mu \mathrm{g} / \mathrm{mL}$ for Ritonavir and 15-75 $\mu \mathrm{g} / \mathrm{mL}$ for Atazanavir respectively.

\section{Procedure for analysis of tablets}

15 tablets were weighed and powdered. Accurately weighed portion of this powder equivalent to 10 $\mathrm{mg}$ of Ritonavir and $10 \mathrm{mg}$ of Atazanavir was transferred to a $100 \mathrm{ml}$ volumetric flaskcontaining 80 $\mathrm{ml}$ of mobile phase. The contents of the flask were allowed to stand for 15 minutes with sonication toensure that to complete solubility of the drugs and make up the volume with mobile phase. The above solution was filtered through $0.45 \mu$ mnylon filter. From this solution appropriate dilutions were made with mobile phase to obtain concentration in calibration range for both the drugs and this solution was used for estimation.With the optimized conditions, a steady baseline was recorded, the mixed working standard solution were injected and the chromatogram was record. The retention times of Ritonavir and Atazanavir were found. The proposed method was found to be specific and no interference from common tablet excipients like starch etc. The response factors of the standard solutions and sample solutions were calculated. The assay was calculated from the equation of regression line for each drug. The assay procedure was repeated for 6 times and the percentage of individual drug in the formulation was calculated. The results of analysis shows that the amount of drug was in good

\section{METHOD VALIDATION}

\section{SYSTEM SUITABILITY}

Accurately weigh and transfer $10 \mathrm{mg}$ of Ritonavir and 10mg of Atazanavir working standard into a $10 \mathrm{ml}$ of clean dry volumetric flasks add about $7 \mathrm{~mL}$ of Diluents and sonicate to dissolve it completely and make volume up to the mark with the same solvent. (Stock solution)

Further pipette $0.15 \mathrm{ml}$ of Ritonavir and $0.45 \mathrm{ml}$ of Atazanavir from the above stock solutions into a $10 \mathrm{ml}$ volumetric flask and dilute up to the mark with diluents.

\section{Procedure:}

The standard solution was injected for five times and measured the area for all five injections in HPLC. The $\%$ RSD for the area of five replicate injections was found to be within the specified limits. 


\section{SPECIFICITY STUDY OF DRUG:}

\section{Preparation of Standard Solution:}

Accurately weigh and transfer $10 \mathrm{mg}$ of Ritonavir and 10mg of Atazanavir working standard into a $10 \mathrm{ml}$ of clean dry volumetric flasks add about $7 \mathrm{~mL}$ of Diluents and sonicate to dissolve it completely and make volume up to the mark with the same solvent. (Stock solution)

Further pipette $0.15 \mathrm{ml}$ of Ritonavir and $0.45 \mathrm{ml}$ of Atazanavir from the above stock solutions into a $10 \mathrm{ml}$ volumetric flask and dilute up to the mark with diluents.

\section{Preparation of Sample Solution:}

Take average weight of the Tablet and crush in a mortar by using pestle and weight $10 \mathrm{mg}$ equivalent weight of Ritonavir and Atazanavir sample into a $10 \mathrm{~mL}$ clean dry volumetric flask and add about $7 \mathrm{~mL}$ of Diluent and sonicate to dissolve it completely and make volume up to the mark with the same solvent.

Further pipette $0.15 \mathrm{ml}$ of Ritonavir and $0.45 \mathrm{ml}$ Atazanavir above stock solution into a $10 \mathrm{ml}$ volumetric flask and dilute up to the mark with diluen

\section{Linearity}

The method was linear in the range of $150-450 \mu \mathrm{g} / \mathrm{mL}$ and $37.5-112.5 \mu \mathrm{g} / \mathrm{mL}$ for ATZ and RIT respectively. Linear regression data was given

\section{Precision}

The precision of the method was demonstrated by inter day and intraday studies. In the intraday studies, solutions of standard and sample were repeated 3times in a day and percent relative standard deviation (\%RSD) was calculated. The intraday \%RSD of Ritonavir and Atazanavir were foundto be 0.54 and 0.8 respectively.In the interday variation studies, injections of standard and sample solutions were made on two days and \%RSD was calculated. The interday \%RSD for Ritonavir and Atazanavir were found to be 0.5 to 0.63 respectively. From the data obtained the developed RP-HPLC method was found to be precise.

\section{Accuracy}

The accuracy of the method was determined by recovery experiments. A known amount of concentration of working standard was added to the fixed concentration of the pre-analyzed tablet solution. Percent recovery was calculated by comparing the area before and after the addition of working standard. Forboth the drugs, recovery was performed in the same way. The recovery studies were performed in 3 times This standard addition method was performed at 50\%, 100\%, 150\%level and the percentage recovery wascalculated. Percent recovery was within the range of 98.2 to 99.6 for Ritonavir and 98.2 to99.6 for Atazanavir that indicates method was accurate.

\section{Limit of detection and limit ofquantification}

The Limit of detection and quantification were calculated using standard deviation of the response and slope of calibration curve. The LOD for Ritonavir and Atazanavir was found to be $0.54 \mu \mathrm{g} / \mathrm{ml}$ and $1.4 \mu \mathrm{g} / \mathrm{mlrespectively.} \mathrm{The} \mathrm{LOQ} \mathrm{is} \mathrm{the} \mathrm{smallest} \mathrm{concentration} \mathrm{of} \mathrm{the} \mathrm{analyte,} \mathrm{which} \mathrm{gives} \mathrm{response}$ that can be accurate. The LOQ was $1.6 \mu \mathrm{g} / \mathrm{ml}$ and $4.4 \mu \mathrm{g} / \mathrm{ml}$ for Ritonavir and Atazanavir respectively.

\section{Robustness}

Robustness of the method was checked by making slight changes in chromatographic conditions like mobile phase ratio, $\mathrm{pH}$ of buffer, flow rate. It was observed that there were no marked changes in chromatograms, which demonstrated that the developed RP-HPLC method is robust.

\section{RESUlTS AND DisCUSSION}

The proposed method was found to be linear in the concentration range of $5-25 \mu \mathrm{g} / \mathrm{mland} 15-75 \mu \mathrm{g} / \mathrm{ml}$ for Ritonavir and Atazanavir. The method was specific since excipients in the formulation did not interferein the estimation of Ritonavir and Atazanavir. Accuracy of the method was indicated by recovery values from 98.2 to 99.6 for Ritonavir and 98.2 to99.6 for Atazanavir. Precision is reflectedby \%RSD values less than 2. The LOD for Ritonavir and Atazanavir was found to be 
M.Sathish Kumar et al.

$0.54 \mu \mathrm{g} / \mathrm{ml}$ and $1.4 \mu \mathrm{g} / \mathrm{mlrespectively} \mathrm{LOQ} \mathrm{was} 1.6 \mu \mathrm{g} / \mathrm{ml}$ and $4.4 \mu \mathrm{g} / \mathrm{ml}$ for Ritonavir and Atazanavir respectively .Validation parameters were summarized

System suitability:

Table. Results of system suitability for Ritonavir

\begin{tabular}{|c|c|c|c|c|c|c|}
\hline S no & Name & Rt & Area & Height & $\begin{array}{c}\text { USP plate } \\
\text { count }\end{array}$ & $\begin{array}{c}\text { USP } \\
\text { Tailing }\end{array}$ \\
\hline 1 & Ritonavir & 2.247 & 136092 & 14051 & 5506 & 1.36 \\
\hline 2 & Ritonavir & 2.246 & 135626 & 14025 & 5674 & 1.2 \\
\hline 3 & Ritonavir & 2.248 & 135557 & 14132 & 5298 & 1.2 \\
\hline 4 & Ritonavir & 2.252 & 136141 & 14306 & 5032 & 1.0 \\
\hline 5 & Ritonavir & 2.248 & 136557 & 14152 & 5812 & 1.33 \\
\hline Mean & & & 135994.6 & & & \\
\hline Std. Dev & & & 410.662 & & & \\
\hline$\%$ RSD & & & 0.3 & & & \\
\hline
\end{tabular}

Table. Results of system suitability for Atazanavir

\begin{tabular}{|c|c|c|c|c|c|c|c|}
\hline S no & Name & Rt & Area & Height & $\begin{array}{c}\text { USP plate } \\
\text { count }\end{array}$ & $\begin{array}{c}\text { USP } \\
\text { Tailing }\end{array}$ & $\begin{array}{c}\text { USP } \\
\text { Resolution }\end{array}$ \\
\hline 1 & Atazanavir & 5.452 & 636065 & 39373 & 5146 & 1.04 & 4.0 \\
\hline 2 & Atazanavir & 5.484 & 633325 & 39429 & 5024 & 1.20 & 4.5 \\
\hline 3 & Atazanavir & 5.491 & 633435 & 39403 & 5167 & 1.2 & 4.3 \\
\hline 4 & Atazanavir & 5.482 & 625113 & 39745 & 5076 & 1.1 & 4.1 \\
\hline 5 & Atazanavir & 5.491 & 633435 & 39403 & 5327 & 1.2 & 4.2 \\
\hline Mean & & & 632274.6 & & & & \\
\hline Std. Dev & & & 4166.895 & & & & \\
\hline$\%$ RSD & & \multicolumn{7}{|c|}{0.6} & & & \\
\hline
\end{tabular}

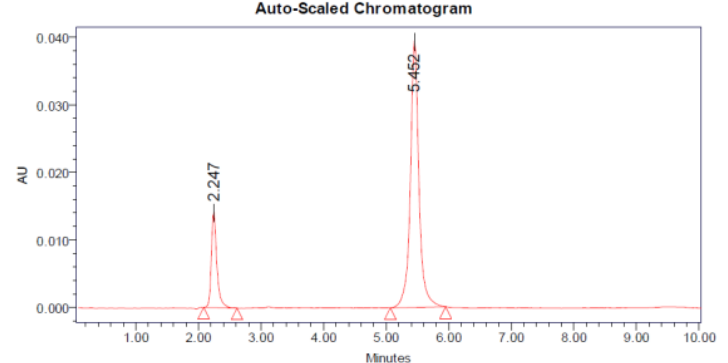

Fig: Chromatogram showing injection -1

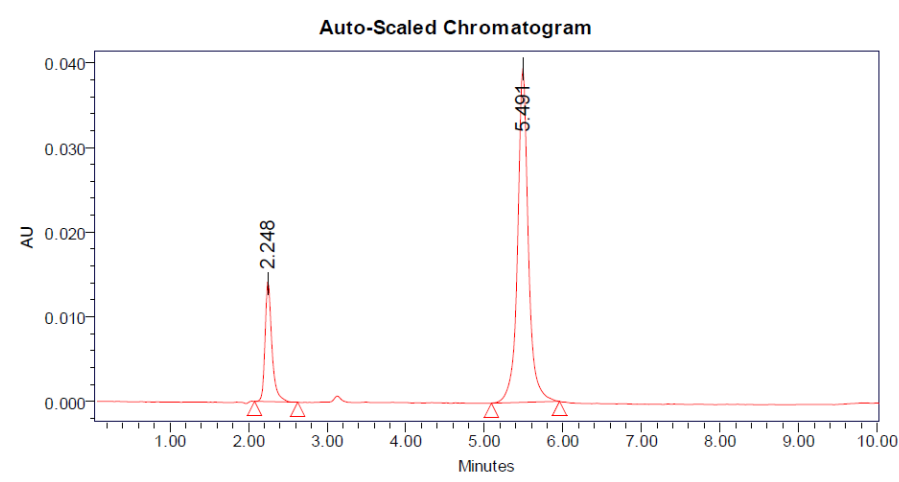

Fig: Chromatogram showing injection -5

\section{SPECIFICITY:}

Table. Peak results for assay standard

\begin{tabular}{|c|c|c|c|c|c|c|c|c|}
\hline Sno & Name & Rt & Area & Height & $\begin{array}{c}\text { USP } \\
\text { Resolution }\end{array}$ & $\begin{array}{c}\text { USP } \\
\text { Tailing }\end{array}$ & $\begin{array}{c}\text { USP } \\
\text { plate } \\
\text { count }\end{array}$ & Injection \\
\hline 1 & Ritonavir & 2.256 & 134994 & 13905 & & 1.32 & 7535 & 1 \\
\hline 2 & Atazanavir & 5.427 & 627906 & 39948 & 4.27 & 1.03 & 5101 & 1 \\
\hline
\end{tabular}


A Validated RP-HPLC Method for the Simultaneous Estimation of Atazanavir and Ritonavir in Pharmaceutical Dosage Forms

\begin{tabular}{|l|c|c|c|c|c|c|c|c|}
\hline \hline 3 & Ritonavir & 2.249 & 136394 & 14163 & & 1.38 & 7701 & 2 \\
\hline 4 & Atazanavir & 5.430 & 636779 & 39935 & 4.13 & 1.05 & 5360 & 2 \\
\hline 5 & Ritonavir & 2.248 & 135870 & 14082 & & 1.40 & 7684 & 3 \\
\hline 6 & Atazanavir & 5.443 & 635760 & 39609 & 4.19 & 1.05 & 5228 & 3 \\
\hline
\end{tabular}

Assay (Sample):

Table. Peak results for Assay sample

\begin{tabular}{|c|c|c|c|c|c|c|c|c|}
\hline Sno & Name & Rt & Area & Height & $\begin{array}{c}\text { USP } \\
\text { Resolution }\end{array}$ & $\begin{array}{c}\text { USP } \\
\text { Tailing }\end{array}$ & $\begin{array}{c}\text { USP } \\
\text { plate } \\
\text { count }\end{array}$ & Injection \\
\hline 1 & Ritonavir & 2.247 & 136092 & 36065 & & 1.26 & 7251 & 1 \\
\hline 2 & Atazanavir & 5.452 & 636779 & 37984 & 4.42 & 1.28 & 5023 & 1 \\
\hline 3 & Ritonavir & 2.246 & 136052 & 33061 & & 1.22 & 7605 & 2 \\
\hline 4 & Atazanavir & 5.461 & 614678 & 39373 & 4.42 & 1.19 & 5146 & 2 \\
\hline 5 & Ritonavir & 2.243 & 134182 & 39537 & & 1.28 & 7228 & 3 \\
\hline 6 & Atazanavir & 5.466 & 635423 & 39457 & 4.48 & 1.20 & 5247 & 3 \\
\hline
\end{tabular}

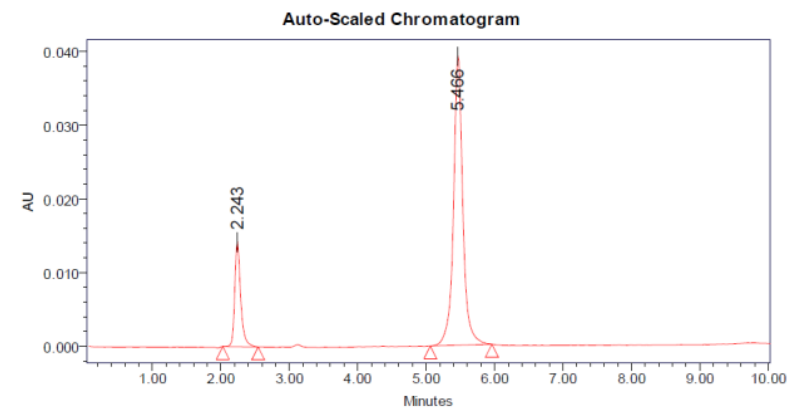

Fig: Chromatogram showing assay of sample injection-3

Assay (Standard):

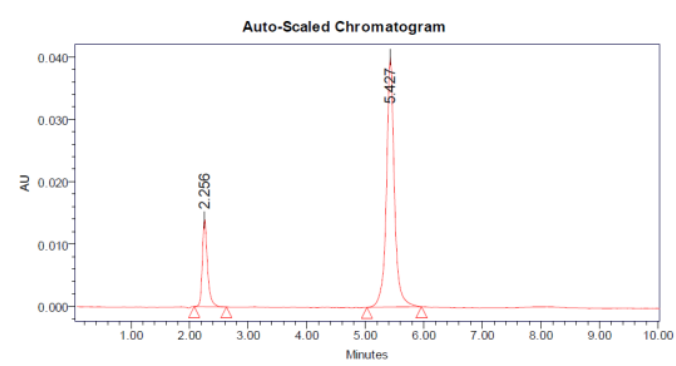

Fig: Chromatogram showing assay of standard injection -1

$\%$ ASSAY =

Sample area Weight of standard Dilution of sample Purity Weight of tablet

$\overline{\text { Standard area }}^{\times} \overline{\text { Dilution of standard }} \times \frac{}{\text { Weight of sample }} \times \frac{100}{100} \times \frac{}{\text { Label claim }} \times 100$

$=628960 / 633481.7 \times 10 / 45 \times 45 / 0.0603 \times 99.7 / 100 \times 2.4122 / 400 \times 100$

$=98.9 \%$

The $\%$ purity of Ritonavir and Atazanavir in pharmaceutical dosage form was found to be $98.9 \%$.

LINEARITY:

Ritonavir:

\begin{tabular}{|c|c|c|}
\hline Concentration Level (\%) & Concentration $\boldsymbol{\mu g} / \mathbf{m l}$ & Average Peak Area \\
\hline 33.3 & 5 & 51080 \\
\hline 66.6 & 10 & 92208 \\
\hline 100 & 15 & 139140 \\
\hline 133.3 & 20 & 180998 \\
\hline 166.6 & 25 & 223920 \\
\hline
\end{tabular}




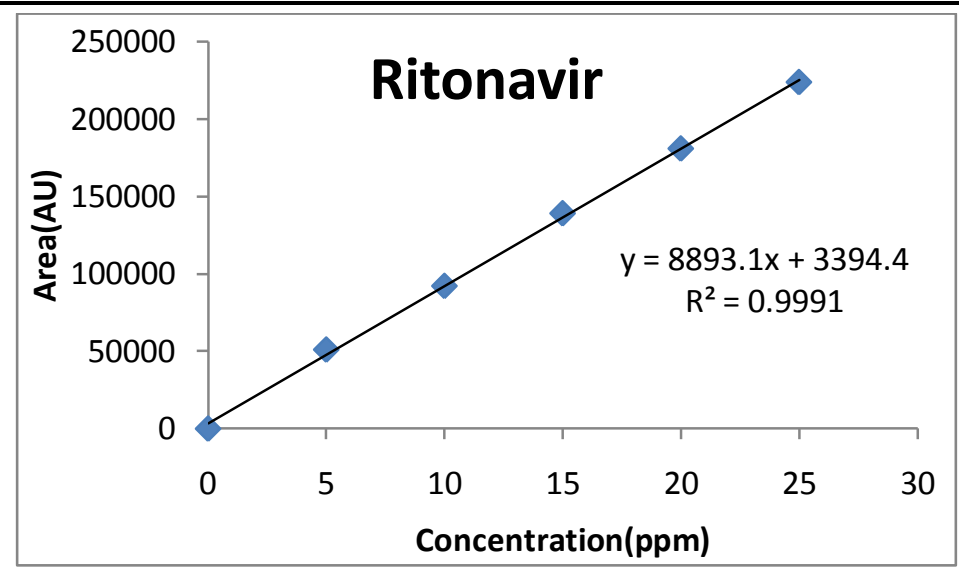

Figure. Calibration graph for Ritonavir

Atazanavir

\begin{tabular}{|c|c|c|}
\hline Concentration Level (\%) & $\begin{array}{c}\text { Concentration } \\
\boldsymbol{\mu g} / \mathbf{m} \mathbf{l}\end{array}$ & $\begin{array}{c}\text { Average } \\
\text { Peak Area }\end{array}$ \\
\hline 33 & 10 & 224573 \\
\hline 66 & 20 & 441895 \\
\hline 100 & 30 & 635379 \\
\hline 133 & 40 & 842226 \\
\hline 166 & 50 & 1041381 \\
\hline
\end{tabular}

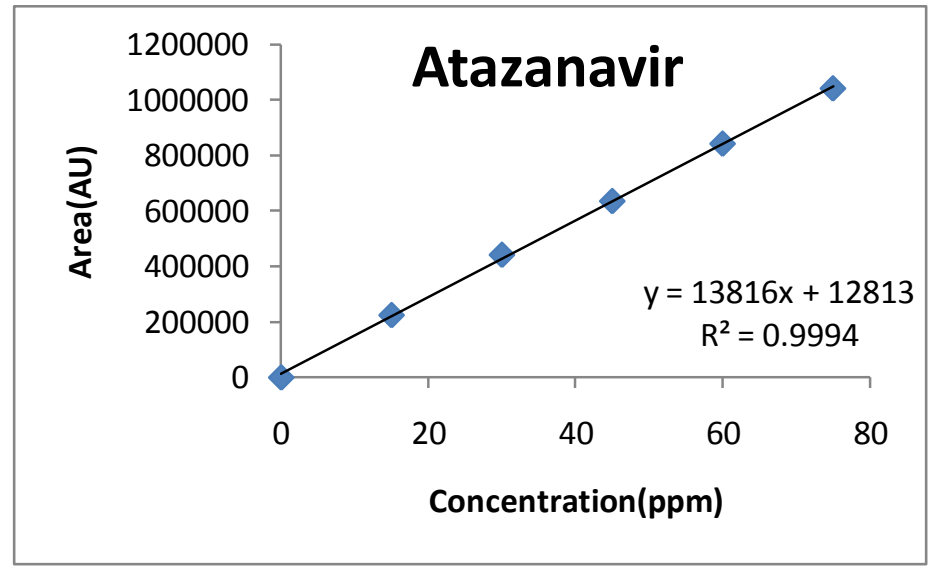

Figure. Calibration graph for Atazanavir

Precision:

Table. Results of repeatability for Ritonavir

\begin{tabular}{|c|c|c|c|c|c|c|}
\hline S no & Name & Rt & Area & Height & $\begin{array}{c}\text { USP plate } \\
\text { count }\end{array}$ & $\begin{array}{c}\text { USP } \\
\text { Tailing }\end{array}$ \\
\hline 1 & Ritonavir & 2.269 & 135148 & 13802 & 7405.7 & 1.2 \\
\hline 2 & Ritonavir & 2.255 & 135369 & 13826 & 7338.4 & 1.2 \\
\hline 3 & Ritonavir & 2.252 & 135451 & 13797 & 7474.5 & 1.19 \\
\hline 4 & Ritonavir & 2.267 & 135812 & 13858 & 7422.2 & 1.18 \\
\hline 5 & Ritonavir & 2.260 & 137007 & 14018 & 7326.6 & 1.21 \\
\hline Mean & & & 135757.4 & & & \\
\hline $\begin{array}{c}\text { Std. } \\
\text { Dev }\end{array}$ & & & 738.3402 & & & \\
\hline$\%$ RSD & & & 0.54 & & & \\
\hline
\end{tabular}

Table. Results of method precession for Atazanavir

\begin{tabular}{|c|c|c|c|c|c|c|c|}
\hline S no & Name & Rt & Area & Height & $\begin{array}{c}\text { USP plate } \\
\text { count }\end{array}$ & $\begin{array}{c}\text { USP } \\
\text { Tailing }\end{array}$ & $\begin{array}{c}\text { USP } \\
\text { Resolution }\end{array}$ \\
\hline 1 & Atazanavir & 5.274 & 630076 & 40629 & 5075.5 & 1.1 & 4.4 \\
\hline
\end{tabular}


A Validated RP-HPLC Method for the Simultaneous Estimation of Atazanavir and Ritonavir in Pharmaceutical Dosage Forms

\begin{tabular}{|c|c|c|c|c|c|c|c|}
\hline \hline 2 & Atazanavir & 5.266 & 630126 & 40937 & 5120.4 & 1.1 & 4.6 \\
\hline 3 & Atazanavir & 5.265 & 632484 & 41279 & 5212.4 & 1.1 & 4.3 \\
\hline 4 & Atazanavir & 5.278 & 636524 & 41454 & 5883.0 & 1.1 & 4.3 \\
\hline 5 & Atazanavir & 5.305 & 621812 & 41320 & 5041.5 & 1.1 & 4.3 \\
\hline Avg & & & 630204.4 & & & & \\
\hline Std. Dev & & & 5375.615 & & & & \\
\hline \%SD & & & 0.8 & & & & \\
\hline
\end{tabular}

Intermediate precision:

Table. Results of Intermediate precision for Ritonavir

\begin{tabular}{|c|c|c|c|c|c|c|}
\hline S no & Name & Rt & Area & Height & $\begin{array}{c}\text { USP plate } \\
\text { count }\end{array}$ & $\begin{array}{c}\text { USP } \\
\text { Tailing }\end{array}$ \\
\hline 1 & Ritonavir & 2.248 & 134029 & 13603 & 7519.3 & 1.2 \\
\hline 2 & Ritonavir & 2.245 & 134202 & 13520 & 7372.9 & 1.2 \\
\hline 3 & Ritonavir & 2.242 & 134745 & 13636 & 7411.8 & 1.19 \\
\hline 4 & Ritonavir & 2.239 & 135442 & 13775 & 7323.5 & 1.20 \\
\hline 5 & Ritonavir & 2.243 & 135535 & 13768 & 7433.4 & 1.23 \\
\hline 6 & Ritonavir & 2.246 & 135699 & 13739 & 7336.9 & 1.3 \\
\hline Mean & & & 134942 & & & \\
\hline Std. Dev & & & 720.3716 & & & \\
\hline \% RS & & & 0.5 & & & \\
\hline
\end{tabular}

Table. Results of Intermediate precision for Atazanavir

\begin{tabular}{|c|c|c|c|c|c|c|c|}
\hline S no & Name & Rt & Area & Height & $\begin{array}{c}\text { USP plate } \\
\text { count }\end{array}$ & $\begin{array}{c}\text { USP } \\
\text { Tailing }\end{array}$ & $\begin{array}{c}\text { USP } \\
\text { Resolution }\end{array}$ \\
\hline 1 & Atazanavir & 5.284 & 636831 & 40102 & 5180.2 & 1.1 & 4.8 \\
\hline 2 & Atazanavir & 5.293 & 638856 & 40464 & 5155.6 & 1.1 & 4.5 \\
\hline 3 & Atazanavir & 5.306 & 630174 & 39977 & 5039.6 & 1.0 & 4.5 \\
\hline 4 & Atazanavir & 5.319 & 630603 & 40748 & 5119.3 & 1.1 & 4.8 \\
\hline 5 & Atazanavir & 5.346 & 632578 & 39772 & 5183.9 & 1.1 & 4.6 \\
\hline 6 & Atazanavir & 5.352 & 636550 & 40083 & 5009.1 & 1.1 & 4.9 \\
\hline Mean & & & 634265.3 & & & & \\
\hline Std. Dev & & & 3629.748 & & & & \\
\hline$\%$ RSD & & & 0.57 & & & & \\
\hline
\end{tabular}

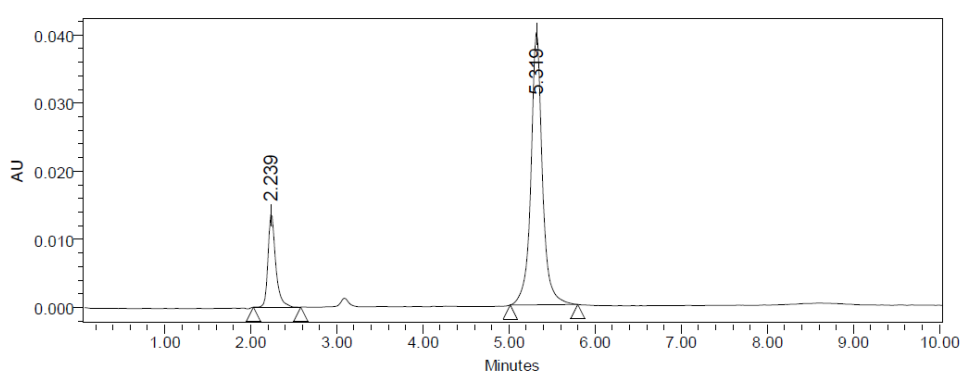

Fig. Chromatogram showing Dayl injection -4

Table. Results of Intermediate precision Day 2 for Ritonavir

\begin{tabular}{|c|c|c|c|c|c|c|}
\hline S no & Name & Rt & Area & Height & $\begin{array}{c}\text { USP plate } \\
\text { count }\end{array}$ & $\begin{array}{c}\text { USP } \\
\text { Tailing }\end{array}$ \\
\hline 1 & Ritonavir & 2.255 & 135442 & 40102 & 7180.2 & 1.2 \\
\hline 2 & Ritonavir & 2.260 & 135535 & 40464 & 7155.6 & 1.20 \\
\hline 3 & Ritonavir & 2.242 & 135699 & 39977 & 7039.6 & 1.1 \\
\hline 4 & Ritonavir & 2.245 & 134657 & 40748 & 7119.3 & 1.2 \\
\hline 5 & Ritonavir & 2.260 & 136754 & 39772 & 7183.9 & 1.24 \\
\hline 6 & Ritonavir & 2.255 & 135908 & 40083 & 7009.1 & 1.3 \\
\hline Mean & & & 135665.8 & & & \\
\hline Std. Dev & & & 682.4683 & & & \\
\hline \%SD & & & 0.5 & & & \\
\hline
\end{tabular}


M.Sathish Kumar et al.

Table. Results of Intermediate precision for Atazanavir

\begin{tabular}{|c|c|c|c|c|c|c|c|}
\hline S no & Name & Rt & Area & Height & $\begin{array}{c}\text { USP plate } \\
\text { count }\end{array}$ & $\begin{array}{c}\text { USP } \\
\text { Tailing }\end{array}$ & $\begin{array}{c}\text { USP } \\
\text { Resolution }\end{array}$ \\
\hline 1 & Atazanavir & 5.266 & 638856 & 39977 & 5039.6 & 1.0 & 4.5 \\
\hline 2 & Atazanavir & 5.265 & 630174 & 40748 & 5119.3 & 1.1 & 4.8 \\
\hline 3 & Atazanavir & 5.306 & 630603 & 39772 & 5183.9 & 1.1 & 4.6 \\
\hline 4 & Atazanavir & 5.293 & 639542 & 40083 & 5009.1 & 1.1 & 4.9 \\
\hline 5 & Atazanavir & 5.265 & 631265 & 56430 & 5023.8 & 1.2 & 4.1 \\
\hline 6 & Atazanavir & 5.266 & 638531 & 47652 & 9123.1 & 1.0 & 4.3 \\
\hline Mean & & & 634828.5 & & & & \\
\hline Std. Dev & & & 4568.678 & & & & \\
\hline \% RD & & & 0.7 & & & & \\
\hline
\end{tabular}

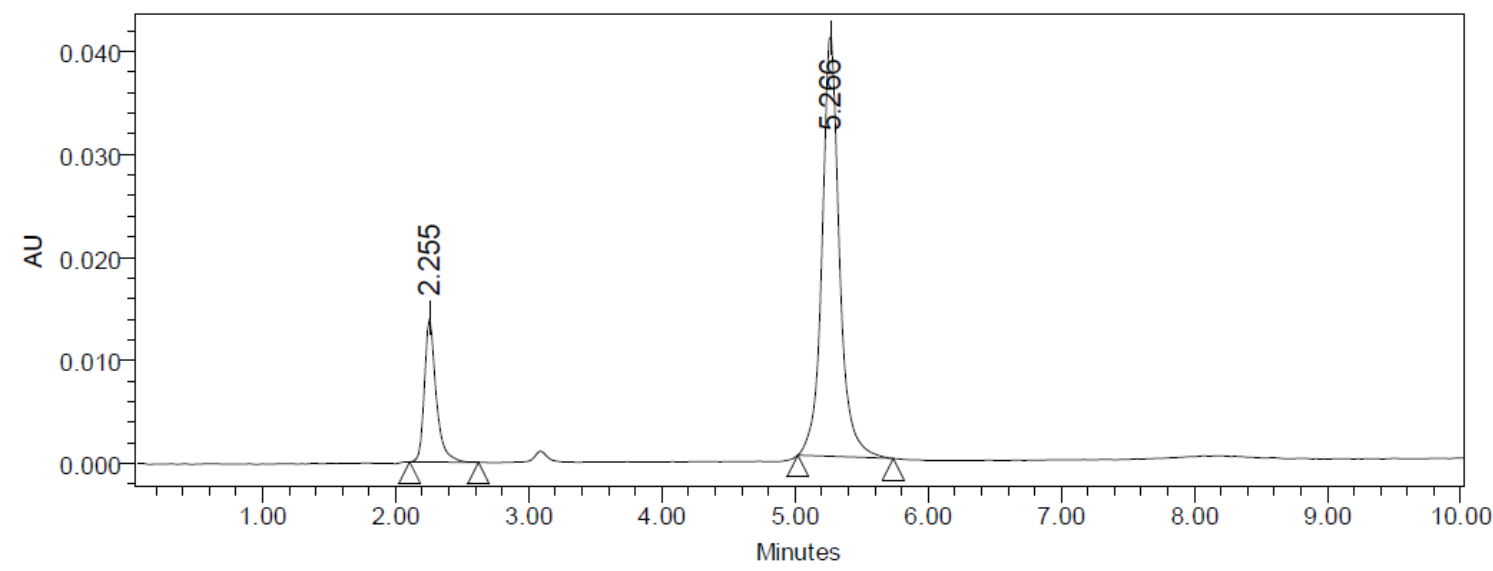

Fig. Chromatogram showing Day 2 injection -6

\section{Accuracy:}

Table. Results of Accuracy for concentration-50\%

\begin{tabular}{|c|c|c|c|c|c|c|c|c|}
\hline Sno & Name & Rt & Area & Height & $\begin{array}{c}\text { USP } \\
\text { Resolution }\end{array}$ & $\begin{array}{c}\text { USP } \\
\text { Tailing }\end{array}$ & $\begin{array}{c}\text { USP } \\
\text { plate } \\
\text { count }\end{array}$ & Injection \\
\hline 1 & Ritonavir & 2.251 & 69955 & 7522 & & 1.30 & 7018 & 1 \\
\hline 2 & Atazanavir & 5.466 & 329816 & 20373 & 4.24 & 1.02 & 5647 & 1 \\
\hline 3 & Ritonavir & 2.251 & 69648 & 7490 & & 1.30 & 7303 & 2 \\
\hline 4 & Atazanavir & 5.447 & 319997 & 20741 & 4.0 & 1.05 & 5001 & 2 \\
\hline 5 & Ritonavir & 2.252 & 69984 & 7553 & & 1.33 & 7000 & 3 \\
\hline 6 & Atazanavir & 5.425 & 319049 & 20762 & 4.92 & 1.02 & 5678 & 3 \\
\hline
\end{tabular}

Table. Results of Accuracy for concentration-100\%

\begin{tabular}{|c|c|c|c|c|c|c|c|c|}
\hline Sno & Name & Rt & Area & Height & $\begin{array}{c}\text { USP } \\
\text { Resolution }\end{array}$ & $\begin{array}{c}\text { USP } \\
\text { Tailing }\end{array}$ & $\begin{array}{c}\text { USP plate } \\
\text { count }\end{array}$ & Injection \\
\hline 1 & Ritonavir & 2.261 & 135064 & 13901 & & 1.34 & 7531 & 1 \\
\hline 2 & Atazanavir & 5.416 & 638215 & 40860 & 4.84 & 1.06 & 5978 & 1 \\
\hline 3 & Ritonavir & 2.261 & 136160 & 14268 & & 1.34 & 7489 & 2 \\
\hline 4 & Atazanavir & 5.395 & 624542 & 40973 & 4.8 & 1.06 & 5029 & 2 \\
\hline 5 & Ritonavir & 2.267 & 135179 & 13716 & & 1.35 & 7366 & 3 \\
\hline 6 & Atazanavir & 5.382 & 633708 & 40591 & 4.87 & 1.07 & 5934 & 3 \\
\hline
\end{tabular}

Table. Results of Accuracy for concentration-150\%

\begin{tabular}{|c|c|c|c|c|c|c|c|c|}
\hline Sno & Name & Rt & Area & Height & $\begin{array}{c}\text { USP } \\
\text { Resolution }\end{array}$ & $\begin{array}{c}\text { USP } \\
\text { Tailing }\end{array}$ & $\begin{array}{c}\text { USP plate } \\
\text { count }\end{array}$ & Injection \\
\hline 1 & Ritonavir & 2.271 & 199987 & 18953 & & 1.40 & 7833 & 1 \\
\hline 2 & Atazanavir & 5.368 & 581475 & 59177 & 4.69 & 1.10 & 5151 & 1 \\
\hline
\end{tabular}


A Validated RP-HPLC Method for the Simultaneous Estimation of Atazanavir and Ritonavir in Pharmaceutical Dosage Forms

\begin{tabular}{|c|c|c|c|c|c|c|c|c|}
\hline \hline 3 & Ritonavir & 2.272 & 199992 & 19231 & & 1.39 & 7907 & 2 \\
\hline 4 & Atazanavir & 5.354 & 582305 & 59272 & 4.62 & 1.11 & 5206 & 2 \\
\hline 5 & Ritonavir & 2.273 & 199949 & 19127 & & 1.39 & 7901 & 3 \\
\hline 6 & Atazanavir & 5.339 & 579831 & 59532 & 4.48 & 1.10 & 5143 & 3 \\
\hline
\end{tabular}

The accuracy results for Ritonavir

\begin{tabular}{|c|c|c|c|c|c|}
\hline $\begin{array}{c}\text { \% Concentration } \\
\text { (at specification } \\
\text { Level) }\end{array}$ & Area & $\begin{array}{c}\text { Amount } \\
\text { Added } \\
\text { (ppm) }\end{array}$ & $\begin{array}{c}\text { Amount } \\
\text { Found } \\
(\mathbf{p p m})\end{array}$ & \% Recovery & $\begin{array}{c}\text { Mean } \\
\text { Recovery }\end{array}$ \\
\hline $50 \%$ & 69862.33 & 7.5 & 7.47 & 99.6 & \multirow{2}{*}{$98.8 \%$} \\
\hline $100 \%$ & 135467.7 & 15 & 14.8 & 98.6 & 98.2 \\
\hline $150 \%$ & 199976 & 22.5 & 22.1 & 9 \\
\hline
\end{tabular}

The accuracy results for Ritonavir

\begin{tabular}{|c|c|c|c|c|c|}
\hline $\begin{array}{c}\text { \% Concentration } \\
\text { (at specification } \\
\text { Level) }\end{array}$ & Area & $\begin{array}{c}\text { Amount } \\
\text { Added } \\
(\mathbf{p p m})\end{array}$ & $\begin{array}{c}\text { Amount Found } \\
(\mathbf{p p m})\end{array}$ & \% Recovery & Mean Recovery \\
\hline $50 \%$ & 69862.33 & 7.5 & 7.47 & 99.6 & \multirow{2}{*}{$98.8 \%$} \\
\hline $100 \%$ & 135467.7 & 15 & 14.8 & 98.6 & \\
\hline $150 \%$ & 199976 & 22.5 & 22.1 & 98.2 & \\
\hline
\end{tabular}

The accuracy results for Atazanavir

\begin{tabular}{|c|c|c|c|c|c|}
\hline $\begin{array}{c}\text { \% Concentration } \\
\text { (at specification } \\
\text { Level) }\end{array}$ & Area & $\begin{array}{c}\text { Amount } \\
\text { Added } \\
\text { (ppm) }\end{array}$ & $\begin{array}{c}\text { Amount } \\
\text { Found } \\
\text { (ppm) }\end{array}$ & \% Recovery & \multirow{2}{*}{$\begin{array}{c}\text { Mean } \\
\text { Recovery }\end{array}$} \\
\cline { 1 - 4 } $50 \%$ & 322954 & 22.5 & 22.47 & 99.3 & \multirow{2}{*}{$99.5 \%$} \\
\hline $100 \%$ & 632155 & 45 & 44.8 & 99.3 & 100 \\
\hline $150 \%$ & 945870.3 & 67.5 & 67.49 & \\
\hline
\end{tabular}

\section{Limit of Detection}

\section{Ritonavir:}

$=3.3 \times 1476.577 / 8893$

$=0.54 \mu \mathrm{g} / \mathrm{ml}$

Atazanavir:

$=3.3 \times 6116.702 / 13816$

$=1.4 \mu \mathrm{g} / \mathrm{ml}$

\section{Limit of Quantitation}

Ritonavir:

$=10 \times 1476.577 / 8893$

$=1.6 \mu \mathrm{g} / \mathrm{ml}$

\section{Atazanavir:}

$=10 \times 6116.702 / 13816$

$=4.4 \mu \mathrm{g} / \mathrm{ml}$

Robustness:

Table. Results for Robustness

Ritonavir:

\begin{tabular}{|c|c|c|c|c|}
\hline Parameter used for sample analysis & Peak Area & Retention Time & $\begin{array}{c}\text { Theoretical } \\
\text { nlates }\end{array}$ & Tailing factor \\
\hline Actual Flow rate of $1.0 \mathrm{~mL} / \mathrm{min}$ & 134994 & 2.256 & 7535 & 1.32 \\
\hline Less Flow rate of $0.9 \mathrm{~mL} / \mathrm{min}$ & 159987 & 2.505 & 7891 & 1.27 \\
\hline More Flow rate of $1.1 \mathrm{~mL} / \mathrm{min}$ & 120653 & 2.046 & 7085 & 1.20 \\
\hline Less organic phase & 149987 & 2.505 & 7098 & 1.20 \\
\hline More organic phase & 120654 & 2.046 & 7123 & 1.27 \\
\hline
\end{tabular}


Atazanavir:

\begin{tabular}{|c|c|c|c|c|}
\hline Parameter used for sample analysis & Peak Area & $\begin{array}{c}\text { Retention } \\
\text { Time }\end{array}$ & Theoretical plates & $\begin{array}{c}\text { Tailing } \\
\text { factor }\end{array}$ \\
\hline Actual Flow rate of $1.0 \mathrm{~mL} / \mathrm{min}$ & 637906 & 5.427 & 5101 & 1.01 \\
\hline Less Flow rate of $0.9 \mathrm{~mL} / \mathrm{min}$ & 657680 & 5.599 & 5407 & 1.03 \\
\hline More Flow rate of $1.1 \mathrm{~mL} / \mathrm{min}$ & 607899 & 4.576 & 5584 & 0.98 \\
\hline Less organic phase & 646750 & 5.599 & 5407 & 1.02 \\
\hline More organic phase & 609025 & 4.576 & 5584 & 0.99 \\
\hline
\end{tabular}

\section{CONCLUSION}

- In the present investigation, a simple, sensitive, precise and accurate RP-HPLC method was developed for the quantitative estimation of Atazanavir and Ritonavir in bulk drug and pharmaceutical dosage forms.

- This method was simple, since diluted samples are directly used without any preliminary chemical derivatisation or purification steps.

- Atazanavir and Ritonavir was freely soluble in ethanol, methanol and sparingly soluble in water.

- Methanol: Water (90:10\% v/v) was chosen as the mobile phase. The solvent system used in this method was economical.

- $\quad$ The $\%$ RSD values were within 2 and the method was found to be precise.

- The results expressed in Tables for RP-HPLC method was promising. The RP-HPLC method is more sensitive, accurate and precise compared to the Spectrophotometric methods.

This method can be used for the routine determination of Atazanavir and Ritonavir in bulk drug and in Pharmaceutical dosage forms.

\section{REFERENCES}

[1] Dr. Kealey and P.J Haines, Analytical Chemistry, $1^{\text {st }}$ edition, Bios Publisher, (2002), PP 1-7.

[2] A.BraithWait and F.J.Smith, Chromatographic Methods, $5^{\text {th }}$ edition, Kluwer Academic Publisher, (1996), PP 1-2.

[3] Andrea Weston and Phyllisr. Brown, HPLC Principle and Practice, $1^{\text {st }}$ edition,

[4] Academic press, (1997), PP 24-37.

[5] Yuri Kazakevich and Rosario Lobrutto, HPLC for Pharmaceutical Scientists, $1^{\text {st }}$ edition, Wiley Interscience A JohnWiley \& Sons, Inc., Publication, (2007), PP 15-23.

[6] Chromatography, (online). URL:http://en.wikipedia.org/wiki/Chromatography.

[7] Meyer V.R. Practical High-Performance Liquid Chromatography, 4 th Ed. England, John Wiley \& Sons Ltd, (2004), PP 7-8.

[8] Sahajwalla CG a new drug development, vol 141, Marcel Dekker Inc., New York, (2004), PP 421-426.

[9] Introduction to Column. (Online),URL:http://amitpatel745.topcities.com/index_files/study/colu mn care.pdf

[10] Detectors used in HPLC (online )URL:http://wiki.answers.com/Q/What_detectors_are_used_in_ HPLC

[11] Detectors (online) ,URL:http://hplc.chem.shu.edu/NEW/HPLC_Book/Detectors/det_uvda.html

[12] Detectors (online) ,URL:http://www.dionex.com/enus/webdocs/64842-31644-02_PDA-100.pdf

[13] Detectors (online),URL:http://www.ncbi.nlm.nih.gov/pubmed/8867705

[14] Detectors (online),URL:http://www.chem.agilent.com/Library/applications/59643559.pdf

[15] Detectors (online),URL:http://hplc.chem.shu.edu/new/hplcbook/detector

[16] Draft ICH Guidelines on Validation of Analytical Procedures Definitions and terminology. Federal Register, vol 60. IFPMA, Switzerland, (1995), PP 1126. 
A Validated RP-HPLC Method for the Simultaneous Estimation of Atazanavir and Ritonavir in Pharmaceutical Dosage Forms

[17] Code Q2B, Validation of Analytical Procedures; Methodology. ICH Harmonized Tripartite Guidelines, Geneva, Switzerland, (1996), PP 1- 8.

[18] Introduction to analytical method validation (online), available from: URL: http://www.standardbase.hu/tech/HPLC\%20validation\%20PE.pdf.

[19] Data elements required for assay validation, (online) available from: URL: http://www.labcompliance.com/tutorial/methods/default.aspx.

[20] Snyder LR practical HPLC method development, $2^{\text {nd }}$ edition. John Wiley and sons, New York, (1997), PP 180-182.

[21] Skoog D A, West D M, Holler FJ: Introduction of analytical chemistry. Sounder college of publishing, Harcourt Brace college publishers. (1994), PP 1-5.

[22] Sharma B K, Instrumental method of chemical analysis Meerut. (1999), PP 175-203.

[23] Breaux J and Jones K: Understanding and implementing efficient analytical method development and validation. Journal of Pharmaceutical Technology (2003), 5, PP 110-114.

[24] Willard, H. y. Merritt L.L, Dean J.A and Settle F.A "Instrumental methods of analysis" $7^{\text {th }}$ edition CBS publisher and distributors, New Delhi, (1991), PP 436-439.

[25] ICH Q2A, "validation of analytical methods, definitions and terminology", ICH Harmonized tripartite guideline, (1999).

[26] Dnyaneshwar Sukhadev Pawar, Manjusha Dole, Sanjay Sawant, Jyoti M Salunke, development and validation of rp-hplc method for the simultaneous Estimation of atazanavir sulphate and ritonavir in bulk and formulations. International Journal of Pharmacy and Pharmaceutical Sciences. Vol 5, Suppl 3, 2013, 905-909

[27] Anusha Tiyyagura, Ashwini Gunda, Annapurna Renee Chitturi, Aravind sai, Method Development And Validation For The Simultaneous Estimation Of Atazanavir And Ritonavir In Pharmaceutical Dosage Form By RP-HPLC. International Journal Of Pharmaceutical, Chemical And Biological Sciences, IJPCBS 2012, 3(1), 44-54.

[28] J. Venkatesh, M. SingaiahChowdary, Haritha, D. Anuroop, V.V.L.N. Prasad and V. Anjani Prasad Reddy, Reverse Phase High Performance Liquid Chromatographic Estimation of Atazanavir and Ritonavirin Pharmaceutical Dosage Form. Global J. Pharmacol., 7 (3): 307-310, 2013.

[29] P. Nagaraju, G. Indira Priyadarshini and SCHVSS. Appaji, Development and Validation of Reverse Phase HPLC Method for the Simultaneous Estimation of Lopinavir and Ritonavir in Pharmaceutical Dosage Forms. International Journal of Research in Pharmaceutical and Biomedical Sciences. Vol. 3 (3) Jul - Sep2012

[30] Ganta Srinivas, Suryadevara Vidyadhara, Ganji Ramanaiah, Srilakshmi V, Method Development and Validation of Stability Indicating RP-HPLC Method for Simultaneous Estimation of Atazanavir and Ritonavir in Bulk and Its Pharmaceutical Formulations. Am. J. PharmTech Res. 2014; 4(4)

[31] Nuli Vasavi, Afroz Patan, Method development and validation for the simultaneous estimation of Atazanavir and Ritonavir in tablet dosage form by RP-HPLC. Indian Journal of Research in Pharmacy and Biotechnology. November - December 2013. Page:808-814. 